\title{
Search for Universal Extra Dimensions in ppbar Collisions
}

\author{
Angelo De Souza Santos* ${ }^{* \dagger}$ \\ DZero Collaboration \\ E-mail: assantosefnal.gov
}

\begin{abstract}
Models that predict the existence of extra spatial dimensions have been studied since the beginning of the 20th century. These models can incorporate gravity in the framework that describes the other interactions and they can present a number of interesting features such as a dark matter candidate. The consequences of the Universal Extra Dimensions (UED) model is explored by searching for the production of Kaluza-Klein particles whose decay chain leads to signature $\mu^{ \pm} \mu^{ \pm}+$jets $+E_{\mathrm{T}}$. Analyzing the data set corresponding to an integrated luminosity of $7.3 \mathrm{fb}^{-1}$, collected by the $\mathrm{D} \emptyset$ detector at a $p \bar{p}$ collider at a center of mass energy of $1.96 \mathrm{TeV}$, no excess was observed and a lower limit was set on the compactification scale of $R^{-1}>260 \mathrm{GeV}$ in the model. This is the first study to impose a direct limit on the minimal UED model.
\end{abstract}

36th International Conference on High Energy Physics

4-11 July 2012

Melbourne, Australia

\footnotetext{
* Speaker.

${ }^{\dagger}$ SPRACE/IFT-UNESP, São Paulo, SP, Brazil
} 


\section{Introduction}

The known universe is composed of fundamental building blocks, fermions and bosons, whose existence is foreseen by a quantum field theory called Standard Model (SM). This is the most sucessful model to describe the interactions of the elementary particles of matter. Concerning the $\mathrm{SM}$, the spontaneous symmetry breaking in the electroweak sector is responsible for giving mass to fermions and gauge bosons, which is possible when including the Higgs potential. Despite a new boson with mass $\sim 125 \mathrm{GeV}$ (similar to the Higgs boson) has been recently observed by ATLAS and CMS collaborations [1,2], crediting more confidence to the Standard Model, it is not expected the SM to be a final theory since there still be some opened questions: there is no quantum description of gravity; no explanation for the amount of baryogenesis observed in the universe; there is no dark matter candidate. In the theoretical point of view, the hierarchy problem of the energy scale between gravitational and eletroweak interactions has been, among others, a motivation to look for Physics beyond the Standard Model.

Many extentions to the Standard Model have been created. Models of Universal Extra Dimensions (UED [3]) are interesting because present candidates to dark matter [4] and have purposes allowing the proton stability [5, 6], incorporating mass to neutinos [6, 7] and unifying the gauge couplings $[8,9]$. The existence of extra dimensions gives rise to KK particles, whose masses are in the order of electroweak scale, opening possibilities of discoveries in the DØ experiment at the Tevatron hadron accelerator.

\section{Universal Extra Dimensions}

The Universal Extra Dimension model allows the existence of spatial extra dimensions with size $R \sim \mathrm{TeV}^{-1}$. In particular, the minimal UED model (MUED [4]) has only one extra dimension, compactified in the orbifold $S_{1} / Z_{2}$. Due to periodic boundary conditions, the KK particles get masses given by $M_{n}^{2}=M_{0}^{2}+\frac{n^{2}}{R^{2}}$, where $M_{0}$ is the mass of the correspondent SM particle and $n=1,2,3, \ldots$ is the excited state. In tree level, the mass spectrum is almost degenerate. This degeneracy is broken with 1-loop radiative corrections [10] and depends on the energy scale $\Lambda$ of the validity of the theory.

The production probability of KK particles is proportional to $R^{-1}$ and interesting phenomenological results appear when $\Lambda>R^{-1}$. The search for signal of extra dimensions presented here works with the first KK excited state $(n=1)$. The haviest particle is the KK gluon $\left(g_{1}\right)$, followed by KK quarks $\left[\mathrm{SU}(2)\right.$ doublet $\left(Q_{1}\right)$ and singlet $\left.\left(q_{1}\right)\right]$, KK gauge bosons $\left(W_{1}^{ \pm}, Z_{1}\right)$, KK leptons [SU(2) doublet $\left(L_{1}\right)$ and singlet $\left.\left(l_{1}\right)\right]$ and KK photon $\left(\gamma_{1}\right)$. The latter is the Lightest KK Particle (LKP) and since it is stable, scapes detection and is identified by detector as a missing transverse energy $\left(\boldsymbol{E}_{\mathrm{T}}\right)$, being a possible candidate for dark matter.

Hadronic decays of the KK particles have the largest branching ratios, but final states from leptonic decays have cleaner tracks and are easier to be studied. A promising channel has from 2 to 4 leptons, jets and $\mathbb{E}_{\mathrm{T}}$ as is examplified in Fig 1. However, due to the mass degeneracy, the leptons have low transverse momentum and some of them may not pass the reconstruction threshold. In this work will be presented a study of a final state with two muons of the same charge, jets and $\boldsymbol{E}_{\mathrm{T}}$. 
Muon tracks are very clean and the requirement of same charge helps rejecting lots of backgrounds.

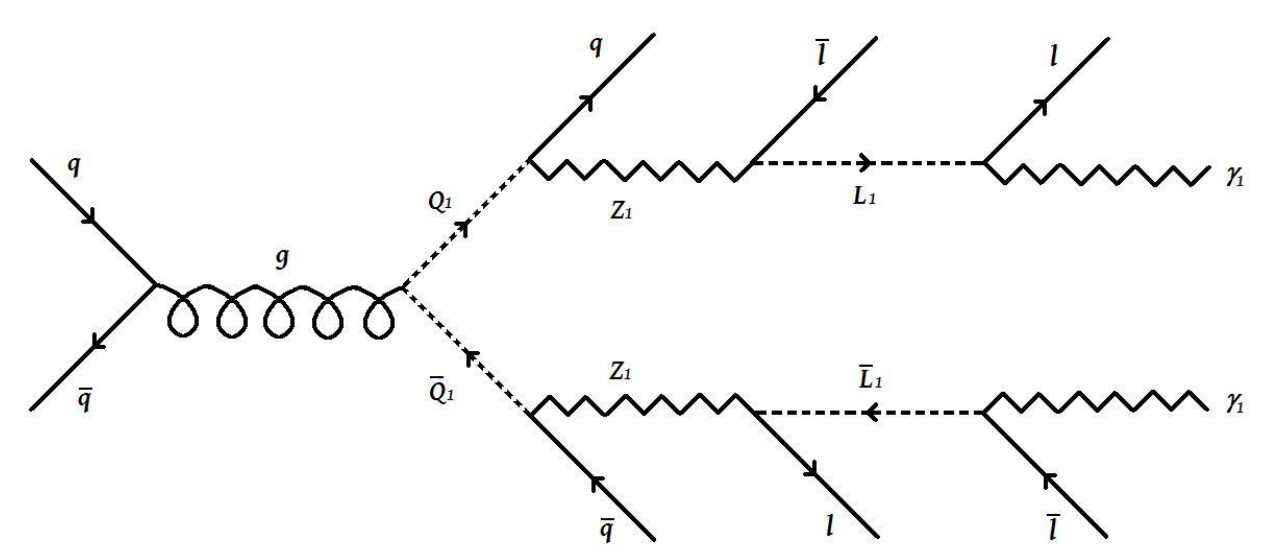

Figure 1: Example of a KK quark pair production followed by a cascade decay through $W_{1}^{ \pm}$and $Z_{1}$ producing jets, 4 leptons and $E_{\mathrm{T}}\left(\gamma_{1}\right)$. Other possible leptonic decay would be either through the $W_{1}^{ \pm} W_{1}^{ \pm}$or $Z_{1} Z_{1}$ pair.

\section{DØ Detector}

Tevatron is a hadron accelarator at Fermilab. With collisions of $1.96 \mathrm{TeV}$ of $p \bar{p}$ center-ofmass energy from April 2002 to September 2011, Tevatron has released $\sim 10 \mathrm{fb}^{-1}$ of integrated luminosity to its two detectors: CDF and DØ. This study presents a search for signal of extra dimensions analyzing $7.3 \mathrm{fb}^{-1}$ of data collected by DØ [11] from April 2002 to July 2010.

$\mathrm{D} \emptyset$ is a hermitic detector of general purpose covering $4 \pi$ geometry space and made of three main sub-systems. Trackings of charged particles are identified in the innermost part of the detector, the tracking system, composed of silicon micro-strips and scintillating fibers, located within a $2 \mathrm{~T}$ solenoid. The tracking system is surrounded by a liquid-argon/uranium calorimeter, where the particle energies are deposited. A cone algorithm of $R=0.5$ [12] is used to reconstruct the jets. Muons are identified in the outermost part of the detector, the muon system, made of a layer of wire chambers in front a $1.8 \mathrm{~T}$ toroid and two layers outside the toroid. Missing transverse energy $\left(E_{\mathrm{T}}\right)$ is measured from the imbalance energy of the detector.

\section{Analysis and Results}

The final state $\mu^{ \pm} \mu^{ \pm}+$jets $+E_{\mathrm{T}}$ is studied based on the MUED model analyzing $7.3 \mathrm{fb}^{-1}$ of integrated luminosity of data collected by $\mathrm{D} \emptyset$ detector. Several backgrounds are compared against data. ALPGEN Monte Carlo (MC) event generator is used to model backgrounds from $W+$ jets, $Z+$ jets and $t \bar{t}$. Effects of showering and hadronization are included with PYTHIA interface. Diboson production $(W W, W Z$ and $Z Z)$ is simulated by PYTHIA. Multijet background is dominant, where the pair of muons with same charge come from heavy flavor $(b \bar{b}$ and $c \bar{c})$ jets and is estimated from data. 
Nine different sets of signal MC events are generated for different size of the extra dimension (from $R^{-1}=200$ to $320 \mathrm{GeV}$ in steps of $15 \mathrm{GeV}$ ) using PYTHIA with $\Lambda=10 \mathrm{TeV}$. Table 1 shows the total production cross section and masses of KK particles for each $R^{-1}$. All KK gluon and quark production modes are included. Approximately $1 \%$ of events have the pair of $\mu^{ \pm} \mu^{ \pm}$after simulating all cascade decay.

\begin{tabular}{ccccccc}
\hline \hline \multirow{2}{*}{$R^{-1}$} & \multicolumn{4}{c}{ Masses $(\mathrm{GeV})$} & \multicolumn{2}{c}{ Cross Section } \\
$(\mathrm{GeV})$ & $\gamma_{1}$ & $Z_{1}$ & $g_{1}$ & $\ell_{1}$ & $Q_{1}$ & $(\mathrm{pb})$ \\
\hline 200 & 201 & 230 & 269 & 207 & 249 & $34.9 \pm 0.2$ \\
215 & 216 & 245 & 287 & 222 & 266 & $20.4 \pm 0.1$ \\
230 & 231 & 260 & 305 & 238 & 283 & $12.1 \pm 0.1$ \\
245 & 246 & 274 & 323 & 253 & 300 & $7.24 \pm 0.05$ \\
260 & 261 & 289 & 341 & 268 & 317 & $4.39 \pm 0.03$ \\
275 & 276 & 304 & 359 & 284 & 334 & $2.69 \pm 0.02$ \\
290 & 291 & 319 & 377 & 299 & 351 & $1.65 \pm 0.01$ \\
305 & 306 & 335 & 395 & 314 & 368 & $1.02 \pm 0.06$ \\
320 & 321 & 350 & 413 & 330 & 385 & $0.63 \pm 0.01$ \\
\hline \hline
\end{tabular}

Table 1: Masses of the KK particles for each $R^{-1}$ used in MC generation with correspondent total cross section. Masses and cross sections are taken from PYTHIA Monte Carlo generator.

All Monte Carlo events pass through the full detector simulation set by GEANT. Effects from pile-up are estimated from data and overlaid with MC events to simulate detector noise and multiple proton-antiproton interactions.

The selection criteria requires events to pass at least one single muon trigger and must contain at least two like-charge muons. Muon tracks in the tracking system must match muon tracks in the muon system in the central region of the detector $(|\eta|<1.5)$. Most of the multijet background is rejected requiring that the transverse momentum of the leading and the next-to-leading muons have $p_{T 1}>15 \mathrm{GeV}$ and $p_{T 2}>10 \mathrm{GeV}$, respectively, as well as avoiding events where muon directions are back-to-back $\left(\Delta_{\mu \mu}>2.9 \mathrm{rad}\right)$. Since multijet background dominates at low $E_{\mathrm{T}}$, events with $E_{\mathrm{T}}<25 \mathrm{GeV}$ are rejected. Events whose muon directions are very close to each other are avoided selecting $\Delta_{\mu \mu}>0.25 \mathrm{rad}$. Cosmic rays are rejected requiring the distance of the closest approach (dca) of the muon tracks to the $p \bar{p}$ interaction vertex (PV) to be $<0.05 \mathrm{~cm}$, and the dca of each muon and PV in the transversal plane $<1 \mathrm{~cm}$. Upper limits in the invariant mass of the muon pair of $M_{\mu \mu}<250 \mathrm{GeV}$ and in the transverse momentum of $p_{T 1}<200 \mathrm{GeV}$ are applied to reduce the number of events where one muon has its charge misreconstructed.

Diboson production is the most similar background to the UED signal when at least one muon come from each gauge boson $\left(W^{ \pm}, Z\right)$. In this case, there is no correlation between muon directions, and no correlation between muon and jet directions as well, identifying a condition where muons are isolated from jets. In the multijet background, however, muons originate from semileptonic meson decays, observing a possible correlation between trajectories of muons and jets. Considering this source of background is not properly modeled by MC genarators, the multijet background is estimated from data.

Therefore, two different samples of data events are defined depending on the muon isolation with respect to jets: the sample $\mathcal{A}$ is composed of two isolated muons and represent the signal 
search region; in the sample $\mathcal{B}$, one of the muons are not isolated. This two samples are divided into sub-regions each based on the transverse momentum of the most isolated muon: a sideband region has $5<p_{T}<10 \mathrm{GeV}$ and a nominal region has $p_{T}>10 \mathrm{GeV}$. Scale factors $\mathscr{N}_{i}$ are calculated dividing the samples $\mathcal{A}$ and $\mathcal{B}$ in the sideband region taking different number of jets into account. Multiplying $\mathscr{N}_{i}$ by sample $\mathcal{B}$ in the nominal region provides an initial estimation $(\mathcal{N})$ of the multijet background. The final estimation arises when other Standard Model process are subtracted from $\mathcal{M}$. Figure 2 shows the data/background comparison (left plot) where there is no clear excess of data.
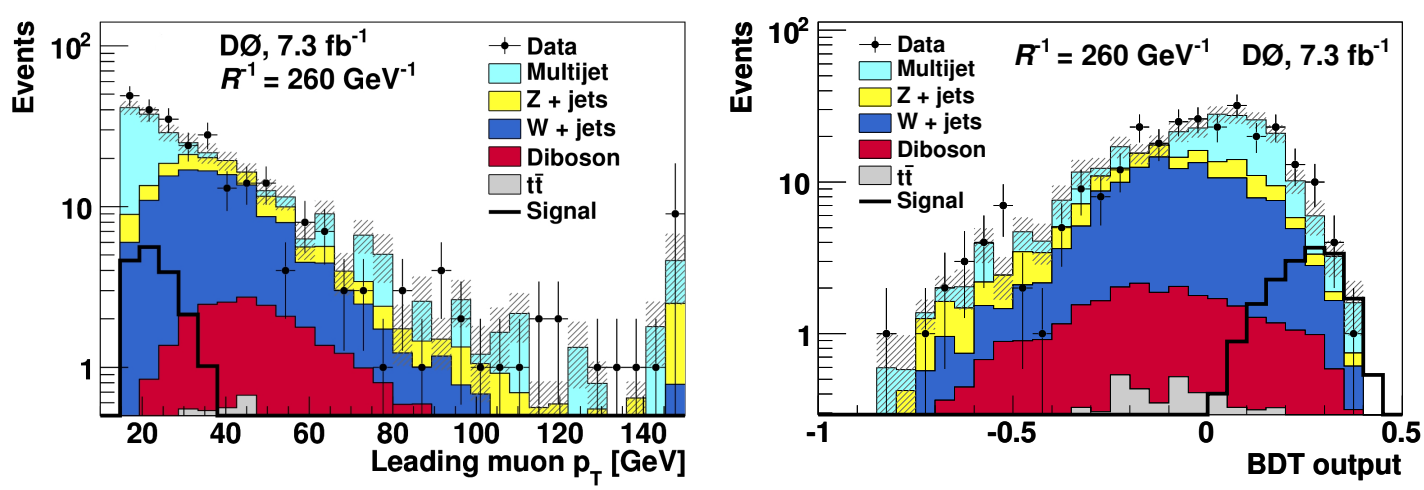

Figure 2: Transverse momentum distribution of the leading muon (left) and output distribution from BDT (right). Data (dots) are compared with all backgrounds (colored histograms). Error bars and hatched areas represent statistical uncertainties for data and backgrounds. The UED signal $\left(R^{-1}=260 \mathrm{GeV}\right)$ is also showed (thick line).

Many different kinematic variables are used to discriminate signal from background. A Tookit for Multivariate Data Analysis (TMVA [13]) is employed and a method called Boosted Decision Tree (BDT) is chosen as the most efficient one to handle those variables and get the best signal optimization against the background rejection. Figure 2 shows the output distribution from BDT (right plot) after taking all kinematic variables as input. Again, no excess of data is observed.

Limits are then set for the UED model. Considering the output from the multivariate approach and the systematic uncertainties, limits are calculated using the $C L_{s}$ method [14] taking the complete shape of the distribution into account to extract the best limit. Observed and expected limits for the cross section of all channels with decays into two muons of same charge as a function of $R^{-1}$ are presented in Fig. 3. The branching ratio $B\left(\mu^{ \pm} \mu^{ \pm}\right)$in Fig. 3 corresponds to the inclusive branching ratio into events with a like-charged muon pair for a given value of $R^{-1}$ in the MUED model. The observed limit crosses the UED cross section at $R^{-1}=260 \mathrm{GeV}$. This point is excluded with $95 \%$ C.L., which translates to a mass of $317 \mathrm{GeV}$ of the lightest KK quark in this model. 


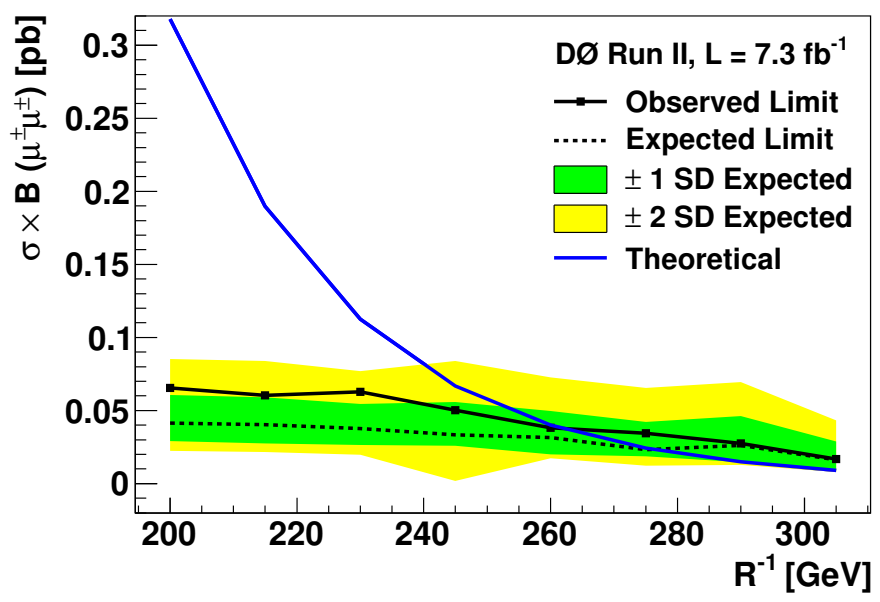

Figure 3: Observed (full black line) and expected (dashed black line) 95\% C.L. limits on $\sigma \times B\left(\mu^{ \pm} \mu^{ \pm}\right.$) as a function of $R^{-1}$. The bands represent \pm 1 (green) and \pm 2 (yellow) standard deviations (SD) around the median expected limits. The expected UED cross section times branching ratio is also showed (blue line).

\section{Conclusion}

This work presents a search for the minimal UED model in the like-sign dimuon channel, using $7.3 \mathrm{fb}^{-1}$. Since there is no excess of data a exclusion limit can be set for the scale $R^{-1} \leq 260 \mathrm{GeV}$ with $95 \%$ confidence level. This is the first direct limit for this model and is published in PRL [15].

\section{References}

[1] G. Aad et al. [Atlas Collaboration], Phys. Lett. B 716 (2012) 1.

[2] S. Chatrchyan et al. [CMS Collaboration], Phys. Lett. B 716 (2012) 30.

[3] T. Appelquist, H.-C. Cheng, B. A. Dobrescu, Phys. Rev. D 64 (2001) 035002.

[4] H.-C. Cheng, K. T. Matchev, M. Schmaltz, Phys. Rev. D 66 (2002) 056006.

[5] T. Appelquist, B. A. Dobrescu, E. Ponton et al., Phys. Rev. Lett. 87 (2001) 181802.

[6] R. N. Mohapatra, A. Perez-Lorenzana, Phys. Rev. D 67 (2003) 075015.

[7] T. Appelquist, B. A. Dobrescu, E. Ponton, et al., Phys. Rev. D 65 (2002) 105019.

[8] K. R. Dienes, E. Dudas, T. Gherghetta, Phys. Lett. B 436 (1998) 55.

[9] K. R. Dienes, E. Dudas, T. Gherghetta, Nucl. Phys. B 537 (1999) 47.

[10] H.-C. Cheng, K. T. Matchev, M. Schmaltz, Phys. Rev. D 66 (2002) 036005.

[11] V. M. Abazov et al. [DØ Collaboration], Nucl. Instrum. Meth. A 565 (2006) 463.

[12] G. C. Blazey, J. R. Dittmann, S. D. Ellis et al., FERMILAB-CONF-00-092-E (2000).

[13] A. Hocker, J. Stelzer, F. Tegenfeldt et al., PoS ACAT (2007) 040.

[14] W. Fisher [DØ Collaboration], FERMILAB Technical Memo. TM-2386-E (2006).

[15] V. M. Abazov et al. [DØ Collaboration], Phys. Rev. Lett. 108 (2012) 131802. 